\title{
anatomy
}

\section{A rare muscle variation - accessory piriformis muscle}

\author{
Albert Gradev, Lina Malinova, Lazar Jelev \\ Department of Anatomy, Histology and Embryology, Medical University of Sofia, Sofia, Bulgaria
}

\begin{abstract}
During routine anatomical dissection of the right lower limb of a 68-year-old male cadaver, a rare muscle variation was revealed and identified as an accessory piriformis muscle. This variant muscle started from the anterior surface of the sacrum below the usual piriformis muscle and extended in a well-identifiable lateral tendon also inserting to the greater trochanter of the femur. In the case described, the accessory piriformis pierced through the proximal part of the sciatic nerve. The length of the additional small muscle was $85 \mathrm{~mm}$ with the broadest part of the muscle belly as $9 \mathrm{~mm}$. The course of the variant muscle, especially its tendinous part, might irritate the sciatic nerve and cause piriformis syndrome and other sciatica-like symptoms. The neurologists who diagnose piriformis syndrome and surgeons performing nerve releasing surgery should be well aware of the described rare muscle variation.
\end{abstract}

Keywords: nerve compression syndrome; piriformis muscle; sciatic nerve; variation

Anatomy 2018;12(3):152-154 @2018 Turkish Society of Anatomy and Clinical Anatomy (TSACA)

\section{Introduction}

The piriformis is a flat, triangular muscle that originates from the anterior surface of the sacrum, and then passes through the greater sciatic foramen to finally insert into the greater trochanter of the femur. ${ }^{[1]}$ Despite its small size and insignificant function as a lateral hip joint rotator, the piriformis has a great importance for the topography of the deep gluteal region. The upper and lower muscle borders form together with the greater sciatic foramen the main foramina for the gluteal neuro-vascular bundles. The piriformis has also special and sometimes variable relations with the largest nerve of the lower limb - the sciatic nerve. ${ }^{[2]}$ Some piriformis-sciatic nerve variations might be related to nerve compression symptoms described as piriformis syndrome. ${ }^{[3]}$ A rare type of piriformis muscle variation is described here that may be a contribution to the understanding of this nerve compression syndrome.

\section{Case Report}

During routine anatomical dissection of the right lower limb of a 68-year-old male cadaver, skin over gluteal region was dissected up to mid-thigh. The gluteus maximus was transected in the middle to expose the deeper structures. Upon dissecting the neurovascular bundles passing through the supra-and infrapiriform foramina, an interesting variation, related to the piriformis muscle and the sciatic nerve was revealed (Figures 1a and $\mathbf{b}$ ). The piriformis muscle presented a complete structure with the usual origin and insertion. As usual, the sciatic nerve emerged from the infrapiriform foramen. There was an interesting additional small muscle identified, that also started from the anterior surface of the sacrum and passed through the greater sciatic foramen below the usual piriformis. The variant muscle presented a well identifiable lateral tendon that pierced through the proximal part of the sciatsic nerve and inserted to the greater trochanter. The length of the additional small muscle was $85 \mathrm{~mm}$ with the broadest part of the muscle belly of $9 \mathrm{~mm}$.

\section{Discussion}

In the literature, there are several variations of the piriformis described - divided piriformis, cleavage of muscle 

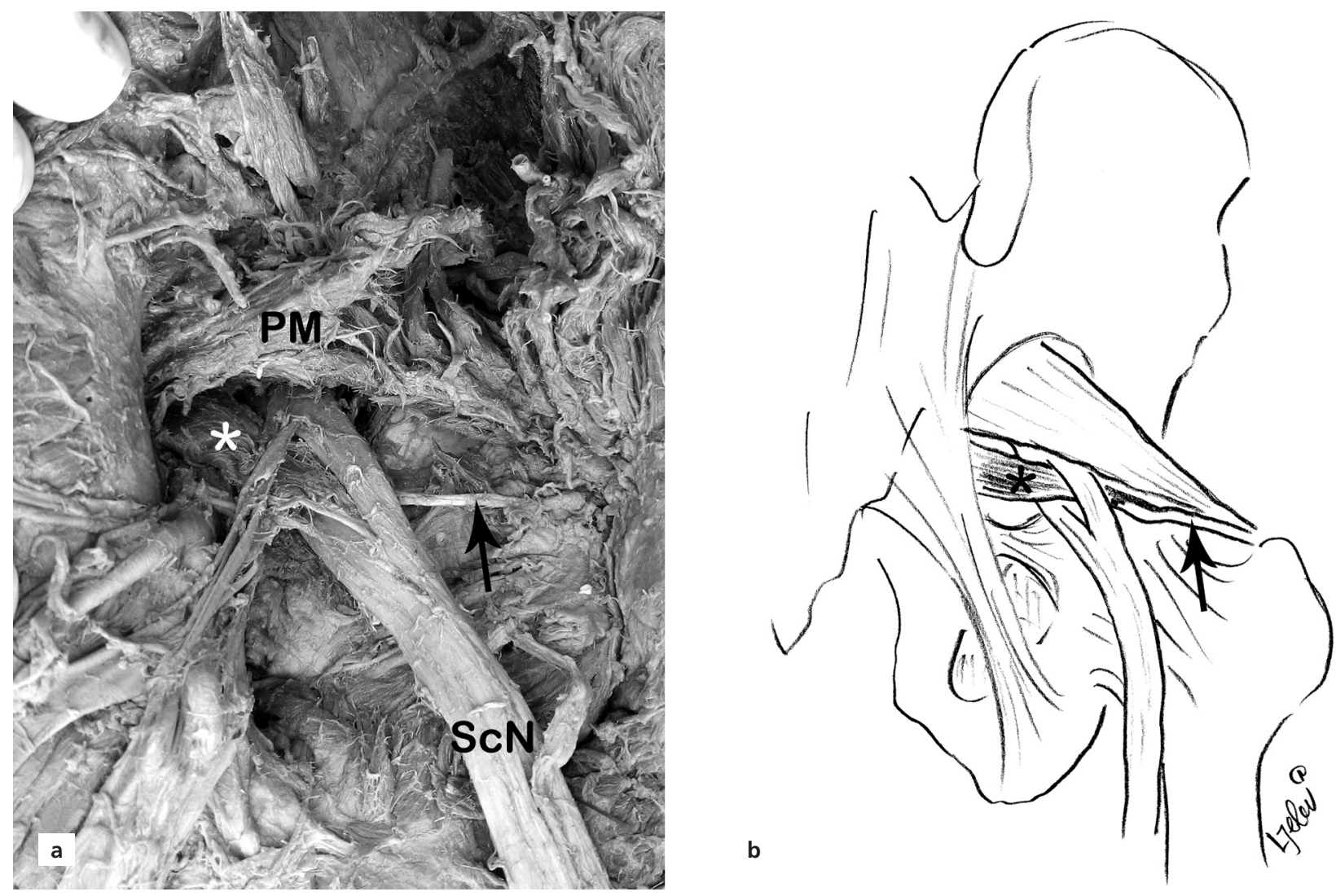

Figure 1. Photograph (a) and schematic diagram (b) of the deep gluteal region. The usual piriformis muscle was partially damaged by the student dissection. The accessory piriformis ${ }^{*}$ ) and its lateral tendon (arrow) passing through the sciatic nerve. PM: piriformis muscle; ScN: sciatic nerve.

bellies, merging with other muscles, or even absence. ${ }^{[4,5]}$ The most important muscle variations, however, are those related to the structure of the proximal sciatic nerve. ${ }^{[6]}$ Six basic types of piriformis-sciatic nerve varitions are described in the literature and their distribution among the human population are presented in details. ${ }^{[7,8]}$

The muscle described here resembles a variation of the piriformis muscle. It is not simply a case of a divided or cleavaged muscle, because it has a separate origin and insertion and distinct proportion of muscle belly to tendon. It can be accepted that this is an accessory muscle. In our case, the sciatic nerve passed below the usual piriformis and was pierced by the accessory piriformis muscle. From a review of the pertinent literature, it seems that the accessory piriformis is quite a rare muscle variation. A similar small muscle below the usual piriformis is also described by Carro et al ${ }^{[9]}$ In their case, however, the accessory piriformis did not penetrate through the sciatic nerve fibers. In two other reports, ${ }^{[10,1]}$ the anomalous sacral attachment of the piriformis with accessory fibers extending over the sacral nerve, seen on MRI, were also described as accessory piriformis muscle.

The reported aberrant muscle might provoke symptoms of compression of the sciatic nerve and be the cause of piriformis syndrome, coccygodynia and following muscle atrophy in the zone innervated by the sciatic nerve. Piriformis syndrome includes hip pain, buttock pain, sciatica and intolerance to sitting. ${ }^{[3,12,13]}$ In up to $16.2 \%$ of surgical case series, piriformis syndrome is caused by anatomical variations. ${ }^{[14]}$ The accessory piriformis, piercing the sciatic nerve, might be one of the rare causes of nerve compression. Therefore, the surgeons who operate in this region must be well aware of the reported muscle variation, as well as the neurologists who diagnose piriformis syndrome and other related sensory symptomatology.

\section{References}

1. Clemente CD. Anatomy of the human body. 30th ed. Philadelphia (PA): Lea and Febiger; 1985. pp. 565-71.

2. Beaton LE, Anson BJ. The relation between the sciatic nerve and of its subdivisions to the piriformis muscle. Anat Rec 1938;70:1-5. 
3. Jankovic D, Peng P, van Zundert A. Brief review. Piriformis syndrome: etiology, diagnosis, and management. Can J Anaesth 2013; 60:1003-12.

4. Bergman RA, Afifi AK, Miyauchi R. Illustrated encyclopaedia of human anatomic variation. Opus I: Muscular system [Revised on January 1, 2019] [Retrieved: March 2019]. Available from: https:// www.anatomyatlases.org/AnatomicVariants/MuscularSystem/Text/ P/24Piriformis.shtml

5. Brenner E, Tripoli M, Scavo E, Cordova A. Case report: absence of the right piriformis muscle in a woman. Surg Radiol Anat 2019 Feb 13. doi: 10.1007/s00276-018-02176-6

6. Natsis K, Totlis T, Konstantinidis GA, Paraskevas G, Piagkou M, Koebke J. Anatomical variations between the sciatic nerve and the piriformis muscle: a contribution to surgical anatomy in piriformis syndrome. Surg Radiol Anat 2014;36:273-80.

7. Adibatti $M$, Sangeetha V. Study on variant anatomy of sciatic nerve. J Clin Diagn Res 2014;8:AC07-9.

8. Sinha MB, Aggarwal A, Sahni D, Harjeet K, Gupta R, Sinha HP. Morphological variations of sciatic nerve and piriformis muscle in gluteal region during fetal period. Eur J Anat 2014;18:2616.

9. Carro LP, Hernando MF, Cerezal L, Navarro IS, Fernandez AA, Castillo AO. Deep gluteal space problems: piriformis syndrome, ischiofemoral impingement and sciatic nerve release. Muscles Ligaments Tendons J 2016;6:384-96.

10. Lee EY, Margherita AJ, Gierada DS, Narra VR. MRI of piriformis syndrome. AJR Am J Roentgenol 2004;183:63-4.

11. Sen A, Rajesh S. Accessory piriformis muscle: an easily identifiable cause of piriformis syndrome on magnetic resonance imaging. Neurol India 2011;59:769-71.

12. Beers MH, Porter RS, Jones TV, Kaplan JL, Berkwits M. The Merck manual of diagnosis and therapy. 18th ed. New Jersey (NJ): Merck Research Laboratories; 2006. p. 2635.

13. Han SK, Kim YS, Kim TH, Kang SH. Surgical treatment of piriformis syndrome. Clin Orthop Surg 2017;9:136-44.

14. Smoll NR. Variations of the piriformis and sciatic nerve with clinical consequence: a review. Clin Anat 2010;23:8-17.

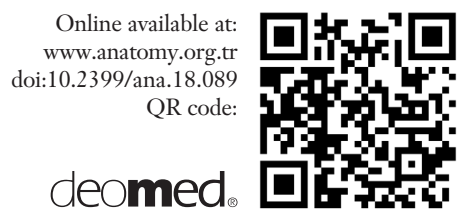

Correspondence to: Lazar Jelev, MD, PhD

Department of Anatomy, Histology and Embryology, Medical University of Sofia, BG-1431, Sofia, Bulgaria

Phone: +359897872751

e-mail: ljelev@abv.bg

Conflict of interest statement: No conflicts declared.

This is an open access article distributed under the terms of the Creative Commons Attribution-NonCommercial-NoDerivs 3.0 Unported (CC BY-NCND3.0) Licence (http://creativecommons.org/licenses/by-nc-nd/3.0/) which permits unrestricted noncommercial use, distribution, and reproduction in any medium, provided the original work is properly cited. Please cite this article as: Gradev A, Malinova L, Jelev L. A rare muscle variation - accessory piriformis muscle. Anatomy 2018;12(3):152-154. 